\title{
THE TIMING OF CHILDBEARING AMONG HETEROGENEOUS WOMEN IN DYNAMIC GENERAL EQUILIBRIUM
}

\author{
Charles H. Mullin \\ Ping Wang \\ Working Paper 9231 \\ http://www.nber.org/papers/w9231 \\ NATIONAL BUREAU OF ECONOMIC RESEARCH \\ 1050 Massachusetts Avenue \\ Cambridge, MA 02138 \\ October 2002
}

The authors have benefited from discussions with Mario Crucini, Mike Grossman, Nezih Guner and Derek Laing. The second author acknowledges financial support from NIH/NIAAA grant no. 1R01-AA11657-01,02. The views expressed herein are those of the authors and not necessarily those of the National Bureau of Economic Research.

(C) 2002 by Charles H. Mullin and Ping Wang. All rights reserved. Short sections of text, not to exceed two paragraphs, may be quoted without explicit permission provided that full credit, including $(\subset$ notice, is given to the source. 
The Timing of Childbearing among Heterogeneous Women in Dynamic General Equilibrium Charles H. Mullin and Ping Wang

NBER Working Paper No. 9231

October 2002

JEL No. J13, J24, D90, D30

\begin{abstract}
We develop a tractable framework with a fully specified dynamic process of demographic and labor decisions over an individual female's life span to determine the timing of childbearing. Fertility affects women's behavior through three channels: its tradeoff with leisure, its interactions with human capital investment, and its cost in terms of lost market productivity. Instead of numerically solving a discrete-time version of the model, we propose an alternative solution technique that provides analytic, closed-form solutions for the continuous-time dynamic optimization problem with (discrete) time-line variables. The analytic results indicate that (i) increased impatience has an ambiguous effect on childbearing timing; (ii) the age at first birth rises at an increasing rate with the productivity loss from children; and (iii) women of greater ability have births at later ages and are more sensitive to parameter changes. Calibration exercises suggest that focusing on the median female's response to changes in the preference, cost, and technology parameters fails to capture their important distributional effects.
\end{abstract}

\author{
Charles H. Mullin \\ Department of Economics \\ Vanderbilt University \\ Nashville, TN 37235 \\ charles.mullin@vanderbilt.edu
}

\author{
Ping Wang \\ Department of Economics \\ Vanderbilt University \\ Nashville, TN 37235 \\ and NBER \\ ping.wang@vanderbilt.edu
}




\section{Introduction}

Fertility choice includes three distinct decisions: the number of children, the quality of children, and the timing of births. A vast literature studies the first two of these aspects of fertility and documents the decline in the total fertility rate over the past century and the accompanying rise in the quality of children. Much less discussed is the timing of fertility, which has undergone changes of the same order of magnitude as those observed in the quantity and quality dimensions. This focus of the literature is not surprising, since the quantity and quality aspects of children can be handled by standard demand and supply analysis with or without dynamics, while the childbearing age requires a fully specified dynamic process of demographic and labor decisions over an individual female's entire life.

As displayed in Figure I, between 1960 and 1997, the median age at first birth in the U.S. increased from 21.8 to 24.2 . This increase is consistent across racial groups, despite a persistent two and a half to three year gap in age at first birth between white and black women. Not surprisingly, Table 1 demonstrates the greater heterogeneity in age at first birth at a point in time relative to the variability in median age across time. In 1997, 93 percent of first births occurred to women aged 15 to 34 , with 55 percent falling between 20 and 29. The lower panel of Table 1 clearly demonstrates the difference in racial groups alluded to earlier. Both black and hispanic women had about twice as many teenage births and slightly more first births in their early twenties in 1997.

In an attempt to analyze the timing of first births, this paper develops a dynamic generalequilibrium continuous-time model for rational individual females over their entire life course with endogenous timing of childbearing. The model considers three important aspects of endogenous fertility, namely, its tradeoff with leisure, its interactions with human capital 
investment, and its cost in terms of productivity loss. ${ }^{1}$ Furthermore, it allows for (ability) heterogeneity and thus provides an explicit account for the distributive effects of preference, cost, and technology parameters on individual behavior and equilibrium outcomes, especially characterizing the distribution of ages at first birth. Notably, our focus on the timing of first births is a good starting point. In particular, the empirical study by Heckman and Walker (1990) using data from the 1981 Swedish Fertility Survey suggests that the strongest effect of economic variables on childbearing timing operates through the time to the first birth.

Traditionally, the solutions to models of this nature cannot be expressed as closed form functions (see Hotz, Klerman and Willis (1997)). Instead, a discrete-time version of the model is specified and solved numerically via backwards recursion methods. A major contribution of this paper is to develop an alternative method for solving models of this kind that provides analytic, closed form solutions. In particular, we solve the model in two steps. First, given an age of childbearing each woman chooses the optimal consumption and human capital investment paths. Second, based on the first-stage solution, she selects the optimal age of childbearing. As previously noted, obtaining an analytic solution to the first-stage optimization problem is extremely difficult. In lieu of discrete-time recursion methods, we derive the hypothetical balanced growth paths of consumption and human capital investment assuming an infinite life span. This solution technique has the advantage of providing analytic solutions, but this advantage is coupled with an inability to address transitional dynamics. In an attempt to compensate partially for the loss of transitional dynamics, a discrete-time version of the model is used both to aid in calibrating the model and to verify the analytic results.

The main findings of our paper are briefly summarized below. The first two results are a product of the general equilibrium, dynamic optimizing framework. First, increased impatience

\footnotetext{
${ }^{1}$ In an empirical study by Millimet (2000), children are found to reduce women's productivity and labor supply, and there is a significant feedback effect from labor to fertility decisions.
} 
for society as a whole reduces time allocated to human capital accumulation but has an ambiguous effect on the timing of childbearing. The former result is a product of the general equilibrium framework, while the latter result, which contrasts sharply to that obtained in partialequilibrium models of family economics, comes from interactions of the former result with dynamic optimization. Second, the age at birth not only rises with the productivity loss from children, but also does so at an increasing rate - this latter finding is an outcome of dynamic optimization in the presence of an endogenous trade-off between childbearing and human capital accumulation.

The remaining findings only can be derived in a model that explicitly accounts for heterogeneity. Perhaps of greatest interest, women of greater ability have births at later ages and are more sensitive to the productivity loss associated with children. Next, stronger peer-group effects reduce the incentive to invest in human capital, which decreases the relative human capital of all women at or above the societal mean level of human capital and raises the age at birth for all women at or below the societal mean level of human capital. Finally, the effects of changes in the preference, cost, and technology parameters on the distributions of childbearing ages are calibrated in the presence of ability heterogeneity. In particular, we find that many of these changes generate asymmetric distributive effects on the timing of childbearing, though some may tighten the distribution and others may widen it.

This research is related to previous studies of demographic transition and economic development. Early studies along these lines focused on predicting fertility for the entire population or explaining differences in fertility across sub-populations (see Spengler and Duncan (1956) and references in the presidential addresses of Lee (1987) and Becker (1988)). This analysis relied heavily on changes in the age, sex, and marital composition of the population, but rarely attempted to formally model the evolution of these inputs. The inability of these models to 
foresee the sharp fertility decline in the early 1930s and the subsequent rise in the 1950s instigated a call for deeper research in this area (see Becker (1960) and Easterlin (1968)). Hotz, Klerman and Willis (1997) provides a comprehensive overview of this more recent literature, so only a few highlights are made here. In particular, fertility became an endogenous variable in growth and macroeconomic models. Barro and Becker (1989) and Becker, Murphy and Tamura (1990) emphasize the interaction of the family with the macroeconomy. Not only does a household's childbearing decision depend on economic conditions, but also these decisions feed back into the economy, influencing labor and capital accumulation decisions. Wang, Yip and Scotese (1994) follow a similar theme, but stress the dynamic interactions between labor, leisure and fertility choices. With the exception of two papers discussed below, a common feature of the endogenous growth and fertility literature is its focus on the quantity-quality tradeoff in fertility decisions. In contrast to these studies, we abstract from the questions of quantity and quality of children, allowing us to isolate the timing of women's first birth.

Conesa (1999) and Caucutt, Guner and Knowles (2001) construct discrete-time general equilibrium models of the timing of fertility. Both of these papers include some aspects of fertility omitted in the current paper, but exclude other aspects considered here. Conesa introduces idiosyncratic uncertainty in future labor earnings and analyzes its impact on fertility decisions by regarding children as irreversible consumption durables. However, he treats the evolution of human capital as exogenous. Caucutt, Guner and Knowles includes marriage and the quantity and quality dimensions of children as endogenous variables. To keep their model tractable, life is divided into five periods in which the latter three one is an adult, but only fertile for the first two of those three intervals. Thus, the timing of birth is reduced to a binary choice. Additionally, the human capital of adults evolves based on time spent in the labor market (i.e., a learning-by-doing rather than an education setup), which eliminates any tradeoff between human 
capital accumulation and market production.

Although the timing of births has not received much attention in the growth literature, the increase in the rate of unwed motherhood over the last thirty years and this population's heavy dependence on government assistance has led to a vast literature on this topic and related issues amongst labor economists. The bulk of this research focuses on the affect of government transfer programs and marital prospects on the fraction of women having teenage births and the marital status of those women at the time of birth (see Hoynes (1997) and Moffitt (1995)). More recently, this line of literature has increased both the choices available to women and the complexity of their utility functions (e.g., Neal (2001), Nycheba (2001), Rosenzweig (1999) and Willis (1999)), but these models continue to share two common traits: (i) fertility decisions are limited to a small number of discrete decisions (e.g., teen versus adult or legitimate versus illegitimate births); and (ii) women optimize in a static environment in which there are no dynamic interactions. In contrast to this literature, our work concentrates on the effects of economic conditions on the commencement of childbearing for all women, not just those at risk for teenage or illegitimate childbearing, and accounts for the dynamic interactions between fertility decisions and other economic factors.

The remainder of the paper is organized as follows. Section 2 describes the economic environment and the first stage individual female's optimization problem. In Section 3, we determine the hypothetical steady-state balanced-growth equilibrium with a hypothetical infinite life span and a given childbearing age. Then, we pin down the age of childbearing given these dynamic paths to complete an individual female's optimization problem. Additionally, we completely characterize human capital investment and birth timing decisions. Section 4 calibrates the model under a given distribution of ability. We begin by examining the responsiveness of the timing of births and the investment in human capital to the underlying 
changes in preference, cost, and technology parameters. Then, we study how these parameters influence the distribution of age at first birth. Finally, Section 5 concludes the paper with a remark concerning possible avenues of future research.

\section{The Model}

The model builds on a continuous-time, perfect-foresight framework with a fertility-consumption tradeoff (in both market and home goods) and a productivity loss from childbearing. The goal of the model is to determine the time at which childbearing begins for women of differing ability, based on their maximizing lifetime utility. In general equilibrium, this structure allows us to characterize the dynamic paths of consumption and human capital investment, as well as the distribution of both these paths and the age at first birth.

Consider a society with a population that is normalized to unity in which each cohort of women is indexed by the age at which they can begin childbearing $(M)$. Each woman seeks to maximize lifetime utility subject to the evolution equations for nonhuman wealth $(b)$ and human capital $(h)$. The lifetime utility up to an exogenous death age $(M+Q)$ is time-separable with a constant time-preference rate $(\rho)$ and an instantaneous utility that is log separable in market goods $(c)$, home goods, and children. Once born, children provide utility of $U_{0}$ and $x$ is an indicator function for the presence of a child. Additionally, children are a (pleasant) distraction who decrease both home and market productivity for $\tau$ years, where $\beta$ and $\phi \in[0,1]$ represent these losses in productivity, respectively.

The solid line in Figure II depicts the actual loss in market productivity, where productivity is normalized to one in the absence of children and, for illustrative purposes, a 20 percent productivity loss has been assumed for the $\tau$ years immediately following a child's birth. This framework creates three distinct states: the years before children, the $\tau$ years in which children affect productivity, and the years after children have matured. To ease the notational 
burden, we replace these three states with two. The years before children are left unchanged, but the years after children are born are collapsed into one state. For an appropriately chosen productivity loss (as depicted by the dashed line if Figure II), the total decrease in lifetime productivity will be identical to the three-state world previously described.

Returning to the optimization problem, each individual divides a unit of time between human capital accumulation (v) and labor supply (1-v). Given the effective wage rate $(w)$ and the real interest rate $(r)$, nonhuman wealth is accumulated from net savings, which is the sum of wages $((1-v)(1-\phi x) w h)$ and interest $(r b)$, net of market expenditures. New investment in human capital is a generalized Cobb-Douglas function of effective-time input $(v(1-\phi x))$, individual human capital and the average human capital stock of her cohort $\left(H_{M}\right)$, where $H_{M}$ represents peer group effects. Human capital is accumulated from new human capital investment net of depreciation $(\eta h)$. Note that children reduce the ability to produce human capital by the same amount as they reduce market production. This symmetry assumption simplifies the analysis and is plausible if the primary reason for a reduction in productivity is lost time due to childrearing.

A representative woman of cohort $M$ solves this intertemporal optimization problem in two steps. First, she chooses the optimal consumption and human capital investment paths for two states: having children as soon as possible ( $x$ always equals one) and never having children ( $x$ always equals zero). To attain an analytic solution for these paths, we derive the hypothetical balanced growth paths of consumption and human capital investment assuming an infinite life span $(Q=\infty)$. Once attained, these paths provide an upper and a lower bound on each woman's consumption based utility. Furthermore, a woman's consumption based utility for any interior childbearing age can be expressed as a weighted average of these two bounds, for the appropriately chosen weights. Second, based on the first-stage solution, she selects the optimal 
age of childbearing. We relegate the second-stage optimization problem (including the determination of the appropriate weights) to the next section, to be handled after the concept of hypothetical balanced growth with a given childbearing status is defined.

The first-stage optimization problem is

$$
\max _{c, v} \int_{M}^{M+Q}\left[\ln c-\beta \mathrm{I}(t \in[M+B, M+B+\tau))+x U_{0}\right] \exp (-\rho(t-M)) d t
$$

subject to

$$
\begin{aligned}
& \dot{b}=r b+(1-v)(1-\phi x) w h-c \\
& \dot{h}=\Gamma v(1-\phi x) h^{\gamma} H_{M}^{1-\gamma}-\eta h,
\end{aligned}
$$

where $\Gamma>0$ is the scaling factor for human-capital production and $1-\gamma \in[0,1)$ indexes the strength of the peer group effect. We normalize $b(0)$ to one. Under exponential discounting with $\rho>0$, the log-linear felicity function in consumption satisfies the Brock-Gale condition automatically, ensuring that both lifetime utility is bounded and the intertemporal optimization problem is well defined. We allow within cohort variation of $\Gamma$, but restrict its distribution to be the same across cohorts and to have compact support.

Except for the inclusion of a productivity loss from childbearing, equation (1) is a prototypical specification of the evolution of nonhuman wealth. In contrast to the human-capital based endogenous growth models in Lucas (1988) and Becker, Murphy and Tamura (1990), however, not only does the loss of productivity from childbearing explicitly enter equation (2), but also the peer-group effect is allowed to affect positively the rate of human capital accumulation. The peer-group effect serves two purposes. First, it ensures that the marginal benefit of investments in human capital for an individual woman is decreasing in the level of investment. Second, it allows for externalities in the accumulation of human capital.

Let $\lambda$ and $\mu$ denote the co-state variables associated with the evolution equations (1) and 
(2), respectively. Let $p \equiv \mu / \lambda$ represent the relative price of human capital investment. Denote the relative human capital stock of an individual of cohort $M$ as $s \equiv h / H_{M}$. Straightforward application of optimal control techniques yield the following two first-order conditions and two Euler equations:

$$
\begin{aligned}
& 1 / c=\lambda \\
& p \Gamma=w s^{1-\gamma} \\
& \dot{\lambda} / \lambda=\rho-r \\
& \dot{\mu} / \mu=\rho+\eta-\Gamma(1-v(1-\gamma))(1-\phi x) s^{\gamma-1}
\end{aligned}
$$

where equation (6) is obtained by substituting equation (4) into the original form of the Euler equation.

Equation (3) specifies intertemporal consumption efficiency. Equation (4) equates the marginal benefit of human capital investment (left-hand side) and the corresponding marginal cost from postponement of current production. Equations (5) and (6) describe the evolution of the shadow prices of market goods and human capital, respectively. While equation (5) is standard in the growth literature, equation (6) deserves further comments. First, the shadow cost of human capital rises when children are present due to the direct loss in productivity. Second, the shadow price increases with the strength of the peer-group effect due to free riding.

We follow the Bond, Wang and Yip (1996) techniques to analyze this generalized twosector endogenous model through both quantities and the relative price of human capital investment, $p$. The incorporation of the relative price greatly simplifies the analysis. Totally differentiating equation (3) and substituting in equation (5) yields the standard Keynes-Ramsey formulation governing the dynamic path of consumption

$$
\dot{c} / c=r-\rho .
$$

Totally differentiating equation (4) implies 


$$
\dot{p} / p=\dot{w} / w+(1-\gamma) \dot{s} / s .
$$

By the definition of $p$, we can combine equations (5) and (6) to obtain

$$
\dot{p} / p=r+\eta-\Gamma(1-v(1-\gamma))(1-\phi x) s^{\gamma-1}
$$

Equation (9) states that if the net rate of returns on nonhuman wealth $(r)$ exceeds the net rate of returns on human capital (the remainder of the right-hand side of equation (9)), then there must be a net capital gain from investment in human capital to maintain no-arbitrage between the two alternative sources of investments. Such a net capital gain from human capital investment is increasing not only in the current level of relative human capital stock, but also in new investment in human capital. Notably, the latter effect is absent without peer groups (i.e., $1-\gamma=$ 0 ). With childbearing, there will be a productivity loss, which requires a compensating increase in the net capital gain from investment in human capital.

In society, the capital market is regarded as exogenous implying a fixed real interest rate. Aggregate female production takes a simple constant-returns-to-scale form in aggregate effective female labor $(L): Y=A L$, where aggregate effective female labor is an integral of all labor supplies from individuals of relevant generation-cohorts:

$$
L=\int_{t-Q}^{t} \int_{i \in M}\left(1-v_{M i t}\right) h_{M i t} d i d M .
$$

This production technology falls within the framework of Rebelo (1991) with his general capital specified as human capital embodied effective labor. Under linear technology, the effective wage is fixed at the constant marginal product of labor,

$$
w=A
$$

Since the effective wage rate is fixed, equation (8) implies that the rate of changes in the relative price of human capital investment must be tied exclusively with the rate of changes in the relative human capital measure. 


\section{Balanced Growth Analysis and Determination of Childbearing Age}

In this section, we begin by defining and characterizing the balanced growth paths of consumption and human capital assuming an infinite life span with a fixed childbearing age. After solving this family of hypothetical paths, we select the childbearing age corresponding to the hypothetical path that maximizes individual welfare.

Our society excludes intergenerational transfers of human capital and wealth. Given zero population growth and a continuum of cohorts, there is no growth in aggregate female output or aggregate effective female labor in the steady state. At the individual level, the hypothetical dynamic paths of consumption and human capital feature perpetual growth.

A balanced growth path is defined as a path such that $c, b$ and $h$ all grow at constant rates, while $v, s$ and $p$ are constant over time. Based on equations (1), (2) and (7), we can verify that along a steady state balanced growth path, consumption, human capital and wealth must grow at the same rate within a generation, which is given by

$$
\dot{c} / c=\dot{b} / b=\dot{h} / h=r-\rho .
$$

For most of the analysis, we focus on a nondegenerate balanced growth path featuring a positive rate of growth. This can be guaranteed by imposing $r>\rho$. This restriction is conventionally referred to as the Jones-Manuelli condition in the growth literature, requiring the rate of return on nonhuman wealth to be sufficiently high to exceed the subjective rate of time discounting. In our application, the above condition is too strong. It is sufficient to assume

\section{Condition H: $r+\eta>\rho$.}

In other words, the flow of human capital cannot fall below the depreciation rate of human capital.

Substituting equation (12) into equations (1) and (2) yields

$$
c / b=\rho+(1-v)(1-\phi x) w(h / b)
$$


and

$$
v=(\eta+r-\rho) s^{1-\gamma} /[\Gamma(1-\phi x)]>0 .
$$

Thus, Condition $\mathrm{H}$ is sufficient to guarantee the fraction of time devoted to human capital investment is positive. To ensure this time allocation is less than unity for any value of $x \in\{0,1\}$, we require: $\max s<[\Gamma(1-\phi x) /(\eta+r-\rho)]^{1 /(1-\gamma)}$. We will return to discuss this latter restriction upon solving for the balanced growth value of $s$.

Combining equations (8), (9) and (14) produces

$$
\dot{s}=\frac{1}{1-\gamma}\left\{s[\eta+r+(1-\gamma)(\eta+r-\rho)]-s^{\gamma} \Gamma(1-\phi x)\right\}
$$

Equation (15) is a constant-coefficient Bernoulli differential equation, which has the general solution

$$
s(t)=\left\{\bar{s}^{1-\gamma}+\left[s_{0}^{1-\gamma}-\bar{s}^{1-\gamma}\right] e^{[r+\eta+(1-\gamma)(\eta+r-\rho)] t}\right\}^{1 /(1-\gamma)}
$$

where $\bar{s}$ is the stationary particular solution ${ }^{2}$

$$
\bar{s}=\left[\frac{\Gamma(1-\phi x)}{\eta+r+(1-\gamma)(\eta+r-\rho)}\right]^{1 /(1-\gamma)} .
$$

Straightforward comparative statics yield:

Proposition 1: Under Condition H, a balanced growth value of relative human capital $\bar{s}$ for each woman with ability $\Gamma$ exists, which possesses the following properties:

(i) it decreases with human capital depreciation, childbearing and the productivity loss associated with children;

(ii) more able women have greater levels of human capital relative to their cohort;

(iii) peer-group effects reduce the relative human capital of all women at or above the cohort

${ }^{2}$ Setting $\dot{s}$ equal to zero and solving for $s$ yields the stationary particular solution of the differential equation, also. 
average level of human capital.

Proposition 1 states that women who produce human capital more easily and women whose productivity loss associated with childbearing is relatively small will have relatively high levels of human capital within their cohort. For an average woman in a cohort $(s=1)$, an increase in the peer group effect $(1-\gamma)$ lowers her relative human capital. More generally, there exists a critical value, $s^{\prime}<1$, such that for all $s<s^{\prime}$ relative human capital rises with the peer group effect, while the relative human capital of those initially above the critical value falls.

Substituting equation (17) into equation (14) yields

$$
v=\frac{\eta+r-\rho}{\eta+r+(1-\gamma)(\eta+r-\rho)} .
$$

Under Condition $\mathrm{H}$, it is not difficult to show that $v \in(0,1)$ without imposing any additional conditions. Straightforward comparative static exercises lead to:

Proposition 2: Under Condition H, a balanced growth allocation of time to human capital investment $v \in(0,1)$ for each woman with ability $\Gamma$ exists, which possesses the following properties:

(i) it increases in the interest rate and the rate of human capital depreciation, decreases in the rate of time preference, and is independent of the productivity loss associated with children;

(ii) peer-group effects reduce the time allocated to human capital investment.

As is typical in a general-equilibrium model, Proposition 2 states that investment in human capital falls as the discount rate $(\rho)$ rises. Also, when the ability to free ride on her peers $(1-\gamma)$ grows, women have less incentive to invest in human capital. Furthermore, investment in human capital increases with both the interest rate and the rate of human capital depreciation 
$(\eta)$. Increases in either of these latter parameters translates into a greater cost of maintaining any given level of human capital stock; the former due to a greater cost in terms of forgone interest and the latter due to a greater fraction of the stock needing replacement. A priori, the effect of increasing the cost of human capital maintenance on the level of investment is ambiguous. The increased cost will cause women to reduce the optimal level of human capital they maintain, but the cost of maintaining any given stock of human capital has increased. Which of these forces dominates could change based on model specifications. However, under the assumption of common growth, the growth rate of human capital is fixed at $r-\rho$, so investment must rise. We now solve for the hypothetical level of consumption at time $0_{+}, \tilde{c}\left(0_{+}\right)$, where the tilde notation represents the hypothetical steady state values for an infinite time horizon and fixed childbearing decision, $\tilde{x}$. Note that changing any parameter of the model except the interest rate net of the discount rate, results in the same hypothetical rate of growth of human capital (equation (12)). So, to model heterogeneity in human capital, we have to create heterogeneity in the initial levels of the human capital stocks. A simple way to accomplish this task is to normalize $h(0)$ to $\Gamma$. This normalization can be combined with equations (13) and (18) to produce

$$
\tilde{c}\left(0_{+}\right)=\rho+(1-\phi \tilde{x}) K A \Gamma
$$

where $K \equiv 1-v$ is a known constant given by equation (18). Since equation (19) solves a hypothetical path for a fixed birth decision, $\tilde{c}\left(0_{+}\right)$is not a function of $B$.

The effect of childbearing on $c\left(0_{+}\right)$is fully characterized by the loss in productivity that accompanies children. As seen in Figure II, we model this productivity loss as a permanent reduction in productivity commencing at the birth of the child and of magnitude $\phi$. To approximate the actual initial level of consumption, we simply replace the step function in productivity induced by children with a smaller productivity loss, $\kappa<\phi$, present throughout 
each woman's entire life (as depicted by the dotted line in Figure II). Under this approximation, we have,

$$
c\left(0_{+}\right)=\rho+(1-\kappa) K A \Gamma .
$$

Let $\kappa(B ; \phi)$ be the function that produces the value of $\kappa$ resulting in the same initial level of consumption as a woman facing a permanent reduction in productivity $\phi$ at time $B$. It is reasonable to assume that $\kappa(B ; \phi)$ is proportional to $\phi$ (by way of approximation) and convex in $B$ (due to market discounting and a finite lifespan). As depicted in Figure III, we approximate $\kappa$ by a woman's discounted average loss in market productivity over her lifespan,

$$
\kappa(B ; \phi)=\phi\left(e^{-\rho B}-e^{-\rho Q}\right) /\left(1-e^{-\rho Q}\right),
$$

where the discount rate $\rho$ is the combination of market discounting at rate $r$ and human capital growth at the rate $r$ minus $\rho .^{3}$ Notably, the signs of the derivatives of $\kappa$, rather than the exact functional form, are critical to the analytic results.

Finally, we are prepared to pin down the timing of childbearing. This is done by first substituting everything back into the lifetime utility to derive the value function,

$$
\begin{aligned}
V & =\int_{0}^{Q}\left[\ln c\left(0_{+}\right)+(r-\rho) t-\beta \mathrm{I}(t \in[M+B, M+B+\tau))+x U_{0}\right] \exp (-\rho t) d t \\
& =\frac{1}{\rho}\left\{V_{0}+\ln c\left(0_{+}\right)\left(1-e^{-\rho Q}\right)+e^{-\rho B}\left[U_{0}\left(1-e^{-\rho(Q-B)}\right)-\beta\left(1-e^{-\rho \min \{Q-B, \tau\}}\right)\right]\right\}
\end{aligned} .
$$

where $V_{0} \equiv(r-\rho)\left[1-(1+\rho Q) e^{-\rho Q}\right] / \rho$ is an integration constant, independent of $B$. Second, take the derivative of equation (22) with respect to $B$ and set it equal to zero, which, for an interior solution, produces:

$$
1=\frac{\left(1-e^{-\rho Q}\right) e^{\rho B}}{\rho\left[U_{0}-\beta\left(1-e^{-\rho \min \{Q-B, \tau\}}\right)\right]} \cdot \frac{\sigma(B)}{B} \equiv J(B),
$$

\footnotetext{
${ }^{3}$ This specification for $\kappa$ slightly understates the loss in productivity both because it ignores the decreased ability to produce human capital and since a finite lifetime decreases the growth rate of human capital.
} 
where $\sigma(B)$ is the elasticity of $c\left(0_{+}\right)$with respect to $B$ given by,

$$
\sigma(B) \equiv \frac{B}{\tilde{c}\left(0_{+}\right)} \frac{\partial \tilde{c}\left(0_{+}\right)}{\partial B}=B \cdot \frac{K A \Gamma}{\rho+(1-\kappa) K A \Gamma} \cdot\left(-\frac{\partial \kappa(B ; \phi)}{\partial B}\right) .
$$

Indeed, we can interpret $J(B)$ as the ratio of the marginal benefit to the marginal cost of delaying childbearing. When $J(B)$ exceeds one over the range from 0 to $Q$, no childbearing occurs, while when $J(B)$ falls below one over this range, childbearing occurs as soon as possible.

In general, we cannot rule out the possibility of multiple equilibria. However, by utilizing (21) and (24), we can rewrite $J(B)$ as:

$$
J(B)=\frac{1}{U_{0}-\beta\left(1-e^{-\rho \min \{Q-B, \tau\}}\right)} \cdot \frac{\phi K A \Gamma}{\rho+\left[1-\phi\left(e^{-\rho B}-e^{-\rho Q}\right) /\left(1-e^{-\rho Q}\right)\right] K A \Gamma} .
$$

Then, it is easily verified that over the range $[0, Q-\tau], J(B)$ becomes monotonically decreasing with a vertical intercept $J_{0}=\phi K A \Gamma /\left\{\rho\left[U_{0}-\beta\left(1-e^{-\rho \tau}\right)\right]\right\}>0$. Therefore, under this parameterization of $\kappa$ a unique solution of $B$ can be obtained (such a solution need not be interior). Figure IV illustrates the three possible solutions: immediate childbearing, an interior solution, and no childbearing.

Straightforward differentiation shows that $J(B)$ is increasing in $\Gamma, A, \phi, \beta$, and $1-\gamma$ and decreasing in $U_{0}$, but the effect of $\rho$ on $J(B)$ is ambiguous (recall that in addition to the relevant discounting terms appearing in (25), $K \equiv 1-v$ depends positively on the time preference rate). By further examining the second derivatives, we can conclude:

Proposition 3: Under Condition H, a balanced growth value of the childbearing age for each woman with ability $\Gamma$ exists and, if it is interior, it possesses the following properties:

(i) it decreases with the valuation of children, increases with the valuation of leisure and an autonomous shift in the effective wage rate, and increases at an increasing rate with the productivity loss from children; 

productivity loss associated with children;

(iii) stronger peer-group effects have an ambiguous effect on the childbearing age in general, but raise the age of birth for all women at or below the average level of human capital; (iv) increased impatience generates an ambiguous effect on the age of births.

It is intuitive that raising the value of children, lowering the preference for leisure or reducing the productivity loss associated with children leads to earlier childbearing. The age at birth is convex in the productivity loss since it reduces earnings both directly and indirectly. There is the direct loss of wages due to decreased productivity, which is compounded by the reduction in human capital, also attributable to decreased productivity. Similarly, women of higher ability face a larger loss in earnings from having children, which causes them to delay childbearing. Furthermore, the convexity of the age at birth with respect to the productivity loss is greater for higher-ability women due to the same compounding effects mentioned above.

Increasing peer-group effects generate two competing forces. First, there is an incentive to decrease time spent investing in human capital due to free riding. This reduction in investment lowers the opportunity cost of children, leading to earlier childbearing. Second, the marginal product of human capital in the production of human capital rises. This second effect causes women to invest more, which increases their human capital and, hence, raises the opportunity cost of childbearing. For women with human capital not exceeding the cohort average, the second effect dominates the first.

There are two direct effects associated with increased impatience. First and perhaps most importantly, it encourages early consumption of all goods (higher $c\left(0_{+}\right)$and earlier childbearing). Second, the present-discounted loss of leisure from childbearing rises with impatience (higher $\beta\left(1-e^{-\rho \tau}\right)$ over the range $B \in[0, Q-\tau]$ ), which generates a greater 
incentive for delaying childbirth. When analyzing the effect of altering the discount rate for a particular woman (a partial equilibrium analysis in which the actions of society are taken as given), these are the only two effects. Only when the first effect dominates the second effect can one conclude that increased impatience reduces the childbearing age.

When the discount rate for society as a whole is changed, there is an indirect generalequilibrium effect; at each point in time, women substitute away from investment in human capital (lower $v$ ) and into current production (higher $K$ ). This substitution interacts with the models dynamics to produce two competing forces with respect to the timing of childbearing. Lower investment in human capital decreases the loss in future productivity associated with childbearing (a smaller stock is effected), thereby inducing earlier births. However, increased market time raises the loss in market production associated with childbearing, raising the marginal benefit of delaying childbirth. Under the assumption of symmetric productivity losses in market and human capital production, it is straightforward (although algebraically tedious) to demonstrate that the net impact of these general equilibrium effects and the first direct effect mentioned above induces earlier childbearing. However, if childbearing has a greater adverse effect on market production relative to human capital production, this result need not hold.

Combining Propositions 1 through 3 establishes the existence of the unique balanced growth equilibrium with common growth in consumption and human capital and with a nondegenerate distribution of age at birth.

\section{Quantitative Analysis}

The quantitative analysis contains three parts. First, we calibrate the model by selecting parameter values consistent with real world observations. Second, we perform sensitivity analysis, varying the key parameter values and looking into their effects on some key endogenous variables, including the age at first birth and society's ability to produce human 
capital. Third, we form a distribution of ability measured by $\Gamma$ and then compute the mean and the standard deviation of the birth date, as well as the minimum and maximum values corresponding to the ability measures.

Although we are unable to solve for the marginal product of human capital in the production of human capital for any particular woman, we can assess the marginal productivity of human capital for society as a whole. In particular, imagine increasing every woman's human capital by a common percentage. Based on equation (2), each woman's productivity in generating human capital is $\Gamma v(1-\phi x) h^{\gamma} H_{M}^{1-\gamma}$, but the derivative of $h^{\gamma} H_{M}^{1-\gamma}$ with respect to a uniform percentage increase in human capital across all women is one. Thus, the marginal product of human capital in the production of human capital for society as a whole is $\Gamma v(1-\phi x)$. As in the previous section, $\phi x$ is approximated by $\kappa(B ; \phi)$. Putting these conditions together implies that the marginal product of human capital is

$$
E \equiv \Gamma v[1-\kappa(B ; \phi)] .
$$

In order to facilitate the quantitative analysis, we adjust $\sigma(B)$, the elasticity of $c\left(0_{+}\right)$ with respect to $B$ described in equation (24), to account for both a finite lifetime and the decreased ability to produce human capital that accompanies childbearing (paralleling the loss in market productivity). To aid in making these adjustments, we numerically solved a discrete-time version of the model via backwards induction in which the state space consisted of the current level of human capital and bonds. The discrete-time version of the wealth and human capital evolution equations (equations (1) and (2)) were substituted directly into the value function, eliminating time devoted to human capital accumulation $(v)$ and consumption $(c) .{ }^{4}$ For

\footnotetext{
${ }^{4}$ The discrete-time model had 50 time periods, each corresponding to one year, 100 possible values for the level of human capital and 200 possible values for the level of bonds (including negative values) for each time period. Upon request, the authors can provide the algorithm used to solve the model (written in $\mathrm{C}++$ ).
} 
illustrative purposes, a representative sample of the optimal paths for human capital and human capital investment attained from the discrete-time model are depicted in Figure V. ${ }^{5}$

As anticipated, a finite lifetime reduces the growth rate of human capital. In particular, the stock of human capital grows early in life, but then decreases later in life. For a wide range of parameter values in the discrete-time model, the optimal path for the human capital stock has an average discounted growth rate of about zero. This decreased growth in the human capital stock alters the approximated loss in productivity associated with childbearing, $\kappa . \kappa$ is defined as a woman's discounted average loss in market productivity over her lifespan, where discounting is done at the market interest rate $r$. Previously, human capital was assumed to grow at the constant rate $r$ minus $\rho$, resulting in a net discount rate of $\rho$. By setting the average growth rate of human capital to zero the net discount rate returns to $r .^{6}$ Thus,

$$
\kappa(B ; \phi)=\phi\left(e^{-r B}-e^{-r Q}\right) /\left(1-e^{-r Q}\right) .
$$

Substituting this expression for $\kappa(B ; \phi)$ into equation (24) yields

$$
\sigma(B)=B \cdot \frac{\phi K A \Gamma}{\rho+(1-\kappa) K A \Gamma} \cdot \frac{r e^{-r B}}{1-e^{-r Q}}
$$

Additionally, the infinite horizon paths evaluated in the steady state ignore the decrease in the ability to produce human capital that accompanies childbearing. It is important to note that the decreased capacity to produce human capital compounds the loss in human capital productivity; not only do women with children receive a lower return on their human capital stock, but also their ability to maintain that stock of human capital decreases. In Figure V, this aspect of fertility can be seen in two ways. First, human capital accumulation peeks a couple of

\footnotetext{
${ }^{5}$ The paths depicted in the graph have been smoothed to eliminate roughness caused by the discrete choice set of savings and human capital levels used to numerical solve the model. ${ }^{6}$ Indeed, we have performed sensitivity analysis allowing the net discount rate to vary above and below the market rate $r$ and found the age of first birth relatively insensitive to such changes.
} 
years before childbearing begins. ${ }^{7}$ Second, after childbearing, investment in human capital is essentially zero until a woman's human capital stock has decreased to the level corresponding to her now lower societal ranking in the ability to produce human capital. The results from the discrete-time version of the model indicate these effects approximately triple the elasticity of initial consumption with respect to age of birth. Therefore, we use an elasticity of initial consumption with respect to $B$ of

$$
\sigma(B)=3 \cdot B \cdot \frac{\phi K A \Gamma}{\rho+(1-\kappa) K A \Gamma} \cdot \frac{r e^{-r B}}{1-e^{-r Q}} .
$$

Under this approximation, $J(B)$, defined in equation (23), remains monotonically decreasing. As a consequence, there is at most one solution to $J(B)=1$ and, when this solution exists, a unique optimal childbearing age.

With regard to the parameterization, childbirth may begin at age $15(M=15$, so women become fertile around age 14) and retirement occurs at age $65(Q=50$, which is not crucial for the purpose of this study on the timing of age at first birth). We set $\tau=15$, that is childrearing takes 15 years (since we hardly observe childbirth after age 50 , we can set $\min \{Q-B, \tau\}=\tau$ in equation (23) under this parameterization). Children reduce productivity 20 percent $(\phi=0.20)$, which is consistent with estimates by Hotz and Miller (1988). ${ }^{8}$ As a result of the two-sector framework with an exogenous capital market, we can focus on one scaling factor (particularly, $\Gamma)$, leaving the other normalized at unity $(A=1)$. Following the conventional growth literature, we select the time preference rate as $\rho=0.04$, the real rate of interest as $r=0.055$ and the human

\footnotetext{
${ }^{7}$ The expected birth of a child (particularly in the near future) lowers the return to current investments in human capital, but has no effect on current market productivity. Thus, as childbearing approaches, women substitute market production for human capital investment, which results in the human capital stock peeking before the child's birth.

${ }^{8}$ Hotz and Miller demonstrate that the true cost is decreasing with the age of the child, but their estimates produce an average loss of about 20 percent per year. Browning (1992) remarks on how this estimate and most other empirical estimates of the time cost of children seem implausibly low.
} 
capital depreciation rate as $\eta=0.05$. More specifically, the selection of a four percent time preference rate is consistent with Kydland and Prescott (1982), while a five percent human capital depreciation rate falls in the estimates from four to nine percent by Heckman (1976). Jones, Manuelli and Rossi (1993) obtains the real interest rate in a world without taxation ranging from five to nine percent, with which our figure of 5.5 percent concurs.

In the absence of good measures of peer-group and leisure cost parameters, we choose the benchmark values as $1-\gamma=0.20$ and $\beta=0.8 U_{0}$. Lacking data for pinning down these three parameters precludes the possibility of a complete calibration exercise. We will partially remedy this problem through checking the sensitivity of our results to perturbations from these benchmarking values. Now, setting the utility from children to $U_{0}=0.6637$ matches the benchmark model to the average of the mean and median age at first birth (24.8 and 24.3, respectively), which is 24.55 . Finally, the mean value of $\Gamma$ is picked at 0.132988 such that the relative human capital $s(x)$ of the average female is unity.

Under the above parameterization, we report (in bold) the benchmark values of the birth date $(B+M)$ and the average-ability measure $(E)$ in Table 2. By construction, childbearing commences at age 24.55. In the benchmark case, the fraction of time devoted to human capital accumulation $(v)$ is 55 percent and the average ability measure is 0.0716 . Our benchmark value of the fraction of time devoted to human capital accumulation is higher than the figure of 41 percent computed by Jones and Manuelli (1993). This discrepancy is not unreasonable given that human capital is the only productive capital in our economy. For the sake of comparison, in the discrete-time model, which accounts for a finite lifetime and the productivity loss associated with children, the faction of time devoted to human capital production decreases throughout a woman's life and averages about 30 percent of her time.

By performing sensitivity analysis, we can study the robustness of the benchmark values 
as well as verify the theoretical comparative-static predictions. First, it can be observed that the average productivity always decreases with impatience. Second, increasing the rate of time preference $(\rho)$ has an ambiguous effect on the timing of births. Within the range 0.2 to 0.45 , when the rate of time preference rises, the desire to substitute early consumption for later consumption dominates the increase in weight placed on lost leisure associated with children, leading to earlier births. However, once the discount rate exceeds approximately 0.045 , the increased weight on lost leisure dominates and the age of childbearing starts to increase sharply. For example, an increase in the time preference rate by only half a percentage point from 0.05 to 0.55 delays childbirth of the median female by more than three years. In general, the effects of time preference on age at birth are asymmetric in the sense that birth dates respond more sensitively when the time preference rate is either low or sufficiently high to approach the real interest rate. Finally, the timing of children is not as sensitive to the time preference rate as human capital decisions. Changes of the time preference rate by 2 percentage points ( 0.04 to 0.02 ) cause women's average productivity to vary by more than 25 percent from the benchmark value, though birth dates only vary by about 2-2.5 years.

Next, an increase in the value of children $\left(U_{0}\right)$ by 20 percent leads to a decrease in birth dates by more than 8.5 years. However, decreases in the value of children have a greater effect on age at birth. Particularly, a reduction in $U_{0}$ by 20 percent causes the median woman to delay childbearing to about age 37, more than 12 years later than the benchmark value. Indeed, a reduction in $U_{0}$ to half of the benchmark level places this age beyond retirement (which certainly exceeds the biological upper bound for fertility). In contrast, society's average ability is relatively insensitive to $U_{0}$. This insensitivity is because (i) $U_{0}$ only affects the lifetime utility and (ii) the productivity loss associated with children enters symmetrically into the production of both human capital and market goods, resulting in no direct intersectoral reallocation effects. 
Similarly, we allow the cost of childrearing $(\phi)$ to range within 20 percent of the benchmark value and find only birth dates are highly responsive to this alternation. An increase in the cost of children delays childbearing. For example, increasing the loss in total productivity to 24 percent during the period of childrearing ( 20 percent above the benchmark) causes a delay in births to about age 35 , which is over 10 years later than the benchmark case. We also consider an autonomous shift in the effective wage rate $(A)$ within a 20 percent range of the benchmark value. While timing decisions are responsive to the wage rate, the response is weaker than that observed with respect to the cost of childrearing. This difference is primarily attributable to the offsetting strong wealth effect associated with a wage change. In particular, an increase in the effective wage rate of 20 percent results in postponing childbearing by approximately 4 years. ${ }^{9}$ Additionally, changes in the relative leisure cost of childbearing $(\beta)$ from 60 to 100 percent of $U_{0}$ cause age at birth to vary by about 6-8 years. Compared to the responses of childbearing ages to the value of children, those with respect to the relative leisure cost are more symmetric.

Finally, due to a free-rider problem, a stronger peer-group effect in the production of human capital (an increase in 1- $\gamma$ ) discourages individual investment in human capital, leading to a delay in childbearing and a detrimental effect on the society's average ability measure. Interestingly, a stronger peer-group effect induces a similar percentage change in woman's birth dates and average ability (about 5 percent in response to a 10 percentage point increase in $1-\gamma$ ). However, a weaker peer-group effect generates a more noticeable change in the average ability than the childbearing age (approximately 5 versus 2.5 percent). This again indicates the

\footnotetext{
${ }^{9}$ Caucutt, Gunner and Knowles (2001) find that even by eliminating the gender gap in wages, the timing of births does not change significantly. Although our result from the alternation in the effective wage rate does not fully correspond to their experiments, a comparison is still informative. The main differences arise because (i) they allow for the substitution of time between leisure and market activities and (ii) they eliminate the trade-off between market production and human capital production (due to their learning-by-doing setup). Both of these differences reduce the sensitivity of birth timing to the wage rate.
} 
asymmetric responses of childbearing decision.

To analyze the effect of changing parameter values on the distribution of outcomes, we specify a symmetric-triangular density function for $\Gamma$ centered at the mean value of $\bar{\Gamma}=0.132988$ and ranging from minus to plus 30 percent of $\bar{\Gamma} \cdot{ }^{10}$ In computations, we approximate this continuous triangle distribution by 15 discrete points at intervals of width 0.005699 , resulting in a standard deviation for $\Gamma$ of 0.00161 . In the benchmark case, the standard deviation of $E$ and $B+M$ are 0.0090 and 2.75, respectively. ${ }^{11}$ Finally, since ability heterogeneity aggregates out, solving the model with a representative agent, as was done in the previous sections, is still valid. ${ }^{12}$

The results in Table 3 suggest that despite a relatively tight distribution with a coefficient of variation around 0.29 (computed based on the mean of $B$ rather than $B+M$ ), age at birth can vary significantly. In particular, birth dates range from 16 to 30 in the benchmark case and from the minimum age (15) to no children over the entire parameter space of considerations. For example, when the value of children decreases to about 55 percent of the benchmark value, the highest ability women no longer desire children at any age. ${ }^{13}$ When the value of children is 20 percent above the benchmark value or when the productivity loss drops by 15 percent from the benchmark value, almost all women have first births as teenagers.

Interestingly, changes in childrearing preference $\left(U_{0}\right)$ and cost $(\phi)$ parameters have

${ }^{10}$ The symmetric-triangle density function with range $[0.7 \bar{\Gamma}, 1.3 \bar{\Gamma}]$ is given by, $f(\Gamma)=(\bar{\Gamma}-|\Gamma-\bar{\Gamma}|) / \int_{0.7 \bar{\Gamma}}^{1.3 \bar{\Gamma}}\left(\bar{\Gamma}-\left|\Gamma^{\prime}-\bar{\Gamma}\right|\right) d \Gamma^{\prime}$.

${ }^{11}$ Note that the 2.75 years standard deviation in age at first birth represents only the variability due to ability heterogeneity, which should be substantially below the total variability in age at first birth.

${ }^{12}$ Heterogeneity in ability does not affect the hypothetical solution method due to both a linear market production technology and the uniform impact of childbearing on human capital and market production. To consider heterogeneity in $\rho$, or any other parameter that fails to aggregate out, would require resolving the model.

${ }^{13}$ According to Vital Statistics data, approximately 18 percent of women in the U.S. never bear children. 
asymmetric "distributive effects" - higher ability women are more sensitive to both of these parameters. The primary causes of this asymmetry are the two compounding effects: (i) the direct effect of ability on the return to human capital investment from current production and (ii) the indirect growth effects of ability on the production of human capital. Thus, more able women's ages at first birth decisions are more sensitive to changes in the parameters. As a result, an increase in the value of children not only lowers the mean birth date, but also results in a tighter distribution of births. Similarly, an increase in the cost of children delays the average birth and widens the spread. In contrast, whenever the effect of increased impatience is to induce earlier births, less able women's childbearing timing decisions are more sensitive to changes in time preference. This is because her marginal benefit of delaying births is relatively flat, thus leading to smaller offsetting forces to the negative impacts of impatience on the age of childbearing. Moreover, since an exogenous change in the effective wage rate generates an income effect, less able women's ages at first births respond more to this change as long as the biological constraint for women to become fertile does not bind.

Finally, the distribution of birth dates tightens as the strength of the peer-group effect (1r) increases, but otherwise it is not too responsive. The former result is due to asymmetric effects of peer-groups on human capital investment; stronger peer-group effects increase human-capital investment for low-ability women and decrease it for high-ability women. This convergence in the levels of human capital investment leads to a convergence in the costs and, hence, timing, of childbearing. The latter result mainly is due to the symmetry in the loss of productivity in the production of both human capital and market goods, which effectively eliminates the first-order interaction term between age at birth and peer-group effects.

\section{Extensions and Concluding Remarks}

Focusing our analysis on the timing of women's first birth has provided several important 
insights for public policy and policy analysis. Before addressing these findings, it is worth mentioning that extending our model to incorporate the traditional quantity-quality tradeoff is straightforward. The utility associated with children, as well as the productivity loss, both currently regarded as constant, can be made increasing functions of both the quantity and the quality of children. After these modifications, the critical decision of when to start childbearing remains qualitatively unchanged. If we further restrict the quantity of children to integers, we can also consider the optimal spacing between births. Childbearing frequency will depend positively on the benefits from increasing returns to scale in home activity and negatively on the increasing opportunity cost of time.

Two important results arise from our endogenous timing childbearing model. First, despite generating substantial increases in the human capital of disadvantaged women, peer group effects in the production of human capital have little impact on these same women's age at childbirth. An important implication of this finding is that if peer groups have a strong influence on teenage childbearing, they likely would operate through social avenues as opposed to direct economic incentives, such as the return to human capital investment.

Second, in the quantitative analysis, depending on the parameter considered, the variability of behavior can be as sensitive, or even more sensitive, to changes in childbearing preference and cost parameter values as the average level of behavior. Thus, concentrating on the mean treatment effect of a parameter change could produce very misleading results for policy analysts, particularly when the population of interest resides primarily in the tails of the distribution. This lends theoretical support to the recent emphasis in the evaluation literature on identifying the distribution of treatment effects, instead of the average treatment effect.

These same findings naturally lead to some extensions of the model. First, a sensitivity analysis with respect to heterogeneity in parameters besides women's ability to produce human 
capital would be interesting. In particular, two prime candidates are the utility derived from children and the productivity loss associated with children. Additionally, the model could be generalized to allow children to differentially affect the production of human capital and market goods. Such a change would increase the sensitivity of the allocation of time between investment in human capital and the production of market goods with respect to all of the parameters considered. Furthermore, breaking this symmetry may have noticeable impacts on the distribution of childbearing ages.

Similarly, allowing for nonseparable utility in leisure and childbearing could also impact the distribution of childbearing ages (while nonseparability in consumption and childbearing may have a stronger potential to alter the results, it is probably unsolvable). Finally, marriage could be incorporated into the model. This would introduce a second time-line variable, which would significantly complicate the analysis. Yet, if an extremely stylized or partial equilibrium marriage market is adopted, we believe that the model could still be analyzed and calibrated. 


\section{References}

Barro, Robert J. and Gary S. Becker. "Fertility Choice in a Model of Economic Growth." Econometrica 57 (March 1989): 655 - 673.

Becker, Gary S. “An Economic Analysis of Fertility,” in NBER (ed.) Demographic and Economic Change in Developed Countries (1960) Princeton, NJ: Princeton University Press.

Becker, Gary S. "Family Economics and Macro Behavior." American Economic Review 78 (March 1988): $1-13$.

Becker, Gary S., Kevin M. Murphy and Robert Tamura. "Human Capital, Fertility, and Economic Growth.” Journal of Political Economy 98 (October 1990): S12 - S37.

Bond, Eric W., Ping Wang and Chong Yip, “A General Two-Sector Model of Endogenous Growth with Human and Physical Capital: Balanced Growth and Transitional Dynamics," Journal of Economic Theory 68 (July 1996): 149 - 173.

Browning, Martin, "Children and Household Economic Behavior,” Journal of Economic Literature 30 (September 1992): 1434 - 1475.

Caucutt, Elizabeth M., Nezih Guner, and John Knowles, "The Timing of Births: A Marriage Market Analysis," manuscript (October 2001).

Conesa, Juan Carlos, "Educational Attainment and Timing of Fertility Decisions," manuscript (July 1999).

Easterlin, Richard A. Population, Labor Force, and Long Swings in Economic Growth. New York: Columbia University Press (1968). 
Heckman, James J., “A Life-Cycle Model of Earnings, Learning, and Consumption,” Journal of Political Economy (August 1976): S11 - S44.

Heckman, James J. and James R. Walker, "The Relationship Between Wage and Income and the Timing and Spacing of Births: Evidence from Swedish Longitudinal Data," Econometrica (November 1990): 1411 - 1441.

Hotz, V. Joseph and Robert A. Miller, “An Empirical Analysis of Life Cycle Fertility and Female Labor Supply,” Econometrica (January 1988): 91 - 118.

Hotz, V. Joseph, Jacob A. Klerman, and Robert J. Willis, “The Economics of Fertility in Developed Countries," in Handbook of Population and Family Economics, ed. by M. R. Rosenzweig and O. Stark. Elsevier Science (1997).

Hoynes, Hilary Williamson. "Does Welfare Play Any Role in Female Headship Decisions?" Journal of Public Economics 65 (1997): 89 - 117.

Jones, Larry E., Rodolfo Manuelli and Peter E. Rossi, "Optimal Taxation in Models of Endogenous Growth,” Journal of Political Economy (June 1993): 485 - 517.

Kydland, Finn E. and Edward C. Prescott, "Time to Build and Aggregate Fluctuations," Econometrica (November 1982): 1345 - 1370.

Lee, Ronald D. "Population Dynamics of Humans and Other Animals." Demography 24 (November 1987): $443-467$.

Millimet, Daniel L., "The Impact of Children on Wages, Job Tenure, and the Division of Household Labour,” Economic Journal 110 (March, 2000): C129 - C157. 
Moffitt, Robert, "The Effect of the Welfare System on Nonmarital Childbearing." In Report to Congress on Out-of-Wedlock Childbearing (1995), National Center for Health Statistics, Washington DC: U.S. Government Printing Office.

Neal, Derek, "The Economics of Family Structure," manuscript (December 2001).

Nechyba, Thomas J., "Social Approval, Values and AFDC: A Re-Examination of the Illegitimacy Debate," Journal of Political Economy 109 (June 2001): 637 - 841.

Lucas, Robert E., Jr., "On the Mechanics of Economic Development," Journal of Monetary Economics 22 (January 1988): $2-42$.

Rebelo, Sergio, “Long-run Policy Analysis and Long-run Growth,” Journal of Political Economy 99 (August 1991): $500-521$.

Rosenzweig, Mark R., "Welfare, Marital Prospects, and Nonmarital Childbearing,” Journal of Political Economy (December 1999): S3 - S32.

Spengler, Joseph J. and Otis D. Duncan. Demographic Analysis: Selected Readings. Glencoe: The Free Press (1956).

Wang, Ping, Chong K. Yip and Carol A. Scotese. "Fertility Choice and Economic Growth: Theory and Evidence." The Review of Economics and Statistics 76 (May 1994): 255 266.

Willis, Robert J., “A Theory of Out-of-Wedlock Childbearing," Journal of Political Economy (December 1999): S33 - S64. 
Table 1: First Births by Age and Race of Mother for the United States in 1997

\begin{tabular}{|l|r|r|r|r|r|r|r|}
\hline \multirow{2}{*}{ Category } & \multicolumn{7}{|c|}{ Percent of Births in the Specified Age Range } \\
\cline { 2 - 8 } & $10-14$ & $15-19$ & $20-24$ & $25-29$ & $30-34$ & $35-39$ & $40+$ \\
\hline \hline All Women & 0.01 & 0.24 & 0.30 & 0.25 & 0.14 & 0.05 & 0.01 \\
\hline White & 0.00 & 0.19 & 0.29 & 0.29 & 0.16 & 0.05 & 0.01 \\
\hline Black & 0.02 & 0.39 & 0.31 & 0.16 & 0.09 & 0.03 & 0.01 \\
\hline Hispanic & 0.01 & 0.33 & 0.35 & 0.19 & 0.08 & 0.03 & 0.01 \\
\hline
\end{tabular}

Note: Approximately two thirds of the births in the 15 to 19 cell occur to women 18 to 19 years old. 
Table 2: Benchmark Equilibrium and Comparative Statics

\begin{tabular}{|c|c|c|c|}
\hline \multirow{2}{*}{\multicolumn{2}{|c|}{$\begin{array}{c}\text { Changes in } \\
\text { (Benchmark in Bold) }\end{array}$}} & \multicolumn{2}{|c|}{ Effects on } \\
\hline & & \multirow{2}{*}{$\frac{B+M}{26.86}$} & \multirow{2}{*}{$\frac{E}{0.0908}$} \\
\hline \multirow{5}{*}{$\rho$} & 0.020 & & \\
\hline & 0.030 & 25.38 & 0.0813 \\
\hline & 0.040 & 24.55 & 0.0716 \\
\hline & 0.050 & 24.98 & 0.0617 \\
\hline & 0.055 & 28.19 & 0.0568 \\
\hline \multirow{5}{*}{$U_{0}$} & $0.8 \cdot 0.6637$ & 36.82 & 0.0725 \\
\hline & $0.9 \cdot 0.6637$ & 30.15 & 0.0721 \\
\hline & $1.0 \cdot 0.6637$ & 24.55 & 0.0716 \\
\hline & $1.1 \cdot 0.6637$ & 19.82 & 0.0711 \\
\hline & $1.2 \cdot 0.6637$ & 15.82 & 0.0705 \\
\hline \multirow{5}{*}{$\phi$} & $0.8 \cdot 0.2$ & 15.00 & 0.0714 \\
\hline & $0.9 \cdot 0.2$ & 18.85 & 0.0714 \\
\hline & $1.0 \cdot 0.2$ & 24.55 & 0.0716 \\
\hline & $1.1 \cdot 0.2$ & 29.88 & 0.0718 \\
\hline & $1.2 \cdot 0.2$ & 34.88 & 0.0720 \\
\hline \multirow{5}{*}{$A$} & $0.8 \cdot 1.0$ & 19.37 & 0.0710 \\
\hline & $0.9 \cdot 1.0$ & 22.17 & 0.0713 \\
\hline & $1.0 \cdot 1.0$ & 24.55 & 0.0716 \\
\hline & $1.1 \cdot 1.0$ & 26.61 & 0.0718 \\
\hline & $1.2 \cdot 1.0$ & 28.40 & 0.0720 \\
\hline \multirow{5}{*}{$\beta$} & $0.6 \cdot U_{0}$ & 18.09 & 0.0708 \\
\hline & $0.7 \cdot U_{0}$ & 21.13 & 0.0712 \\
\hline & $0.8 \cdot U_{0}$ & 24.55 & 0.0716 \\
\hline & $0.9 \cdot U_{0}$ & 28.41 & 0.0720 \\
\hline & $1.0 \cdot U_{0}$ & 32.76 & 0.0723 \\
\hline \multirow{5}{*}{$1-\gamma$} & 0.10 & 23.88 & 0.0756 \\
\hline & 0.15 & 23.76 & 0.0735 \\
\hline & 0.20 & 24.55 & 0.0716 \\
\hline & 0.25 & 25.26 & 0.0698 \\
\hline & 0.30 & 25.90 & 0.0680 \\
\hline
\end{tabular}

Notes: Benchmark values of other parameters are: $Q=50, M=\tau=15$, $A=1, r=0.055, \eta=0.05$ and $\Gamma=0.132988$. The case of "no children" $(B+M>65)$ occurs whenever $U_{0}$ is as low as half of the benchmark value, $\phi$ is as high as twice of the benchmark value, or $\beta$ is as high as 1.5 times the benchmark value. Whenever the interior solution of $B+M$ is below 15 , the corner solution of 15 is applied. 
Table 3: Distribution Effects

\begin{tabular}{|c|c|c|c|c|c|}
\hline \multirow{2}{*}{\multicolumn{2}{|c|}{$\begin{array}{c}\text { Changes in } \\
\text { (Benchmark in Bold) }\end{array}$}} & \multicolumn{4}{|c|}{ Effect of } \\
\hline & & \multirow{2}{*}{$\begin{array}{c}\begin{array}{c}\text { Mean } \\
\text { of } B+M\end{array} \\
26.75 \\
\end{array}$} & \multirow{2}{*}{$\begin{array}{c}\begin{array}{c}\text { Standard } \\
\text { Deviation } \\
\text { of } B+M\end{array} \\
1.13\end{array}$} & \multirow{2}{*}{$\begin{array}{c}\begin{array}{c}\text { Minimum } \\
\text { Value of } \\
B+M\end{array} \\
23.26\end{array}$} & \multirow{2}{*}{$\begin{array}{c}\begin{array}{c}\text { Maximum } \\
\text { Value of } \\
B+M\end{array} \\
29.03\end{array}$} \\
\hline \multirow{5}{*}{$\rho$} & 0.020 & & & & \\
\hline & 0.030 & 25.21 & 1.71 & 19.97 & 28.71 \\
\hline & 0.040 & 24.31 & 2.75 & 16.05 & 29.98 \\
\hline & 0.050 & 24.87 & 5.63 & 15.00 & 38.07 \\
\hline & 0.055 & -- & -- & 15.00 & no children \\
\hline \multirow{5}{*}{$U_{0}$} & $0.8 \cdot 0.6637$ & 36.55 & 3.00 & 27.47 & 42.70 \\
\hline & $0.9 \cdot 0.6637$ & 29.90 & 2.88 & 21.22 & 35.82 \\
\hline & $1.0 \cdot 0.6637$ & 24.31 & 2.75 & 16.05 & 29.98 \\
\hline & $1.1 \cdot 0.6637$ & 19.65 & 2.61 & 15.00 & 25.01 \\
\hline & $1.2 \cdot 0.6637$ & 16.35 & 2.49 & 15.00 & 20.77 \\
\hline \multirow{5}{*}{$\phi$} & $0.8 \cdot 0.2$ & 15.17 & 0.45 & 15.00 & 17.76 \\
\hline & $0.9 \cdot 0.2$ & 18.76 & 2.38 & 15.00 & 24.10 \\
\hline & $1.0 \cdot 0.2$ & 24.31 & 2.75 & 16.05 & 29.98 \\
\hline & $1.1 \cdot 0.2$ & 29.63 & 2.83 & 21.10 & 35.47 \\
\hline & $1.2 \cdot 0.2$ & 34.62 & 2.90 & 25.86 & 40.59 \\
\hline \multirow{5}{*}{$A$} & $0.8 \cdot 1.0$ & 19.28 & 2.72 & 15.00 & 25.41 \\
\hline & $0.9 \cdot 1.0$ & 21.92 & 2.87 & 15.00 & 27.89 \\
\hline & $1.0 \cdot 1.0$ & 24.31 & 2.75 & 16.05 & 29.98 \\
\hline & $1.1 \cdot 1.0$ & 26.37 & 2.63 & 18.44 & 31.78 \\
\hline & $1.2 \cdot 1.0$ & 28.17 & 2.51 & 20.54 & 33.32 \\
\hline \multirow{5}{*}{$\beta$} & $0.6 \cdot U_{0}$ & 18.08 & 2.19 & 15.00 & 23.18 \\
\hline & $0.7 \cdot U_{0}$ & 20.91 & 2.62 & 15.00 & 26.39 \\
\hline & $0.8 \cdot U_{0}$ & 24.31 & 2.75 & 16.05 & 29.98 \\
\hline & $0.9 \cdot U_{0}$ & 28.15 & 2.84 & 19.60 & 34.01 \\
\hline & $1.0 \cdot U_{0}$ & 32.50 & 2.93 & 23.65 & 38.52 \\
\hline \multirow{5}{*}{$1-\gamma$} & 0.10 & 22.64 & 2.84 & 15.00 & 22.64 \\
\hline & 0.15 & 23.52 & 2.79 & 15.15 & 23.52 \\
\hline & 0.20 & 24.31 & 2.75 & 16.05 & 29.98 \\
\hline & 0.25 & 25.02 & 2.71 & 16.87 & 25.02 \\
\hline & 0.30 & 25.66 & 2.67 & 17.61 & 25.66 \\
\hline
\end{tabular}

Notes: $\Gamma$ follows a symmetric-triangular density function over the interval [0.093092, 0.172884], 30 percent below and above the mean 0.132988. The standard error of $\Gamma$ is 0.0161 . In computation, we approximate the continuous triangle distribution by 15 discrete points of identical width 0.005699 . 
Figure I

Median Age at First Birth Over Time and by Race

(Based on child's race until 1980 and mother's race from that point forward)

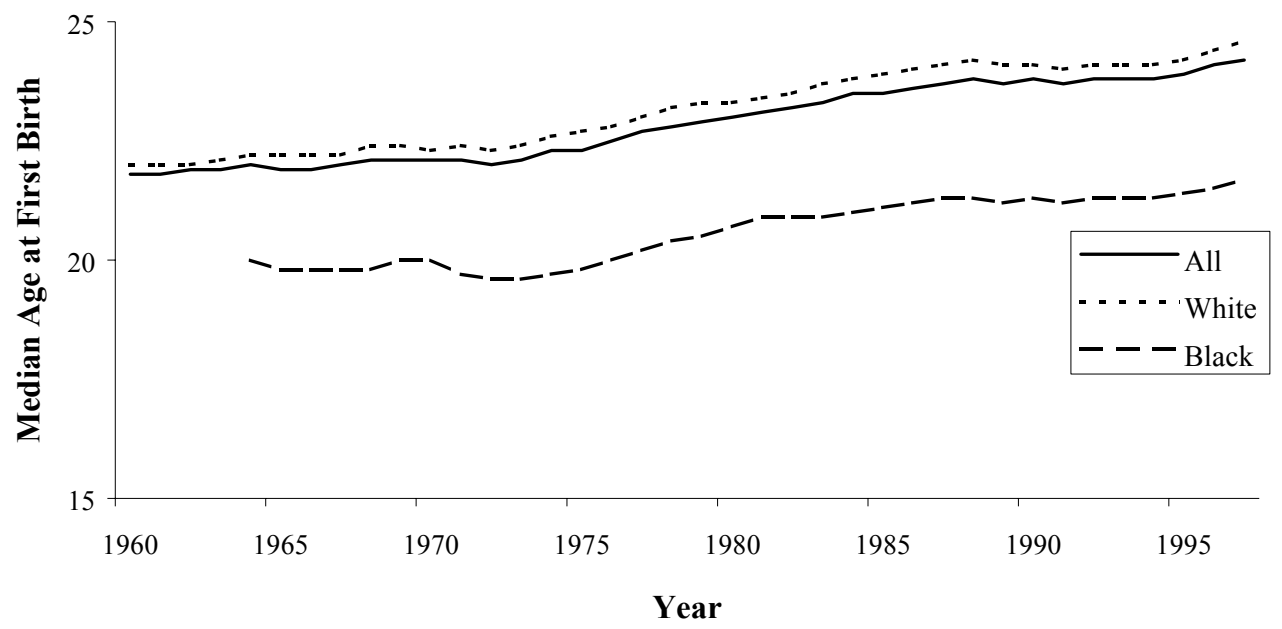

Figure II

Lifetime Productivity as a Function of Fertility

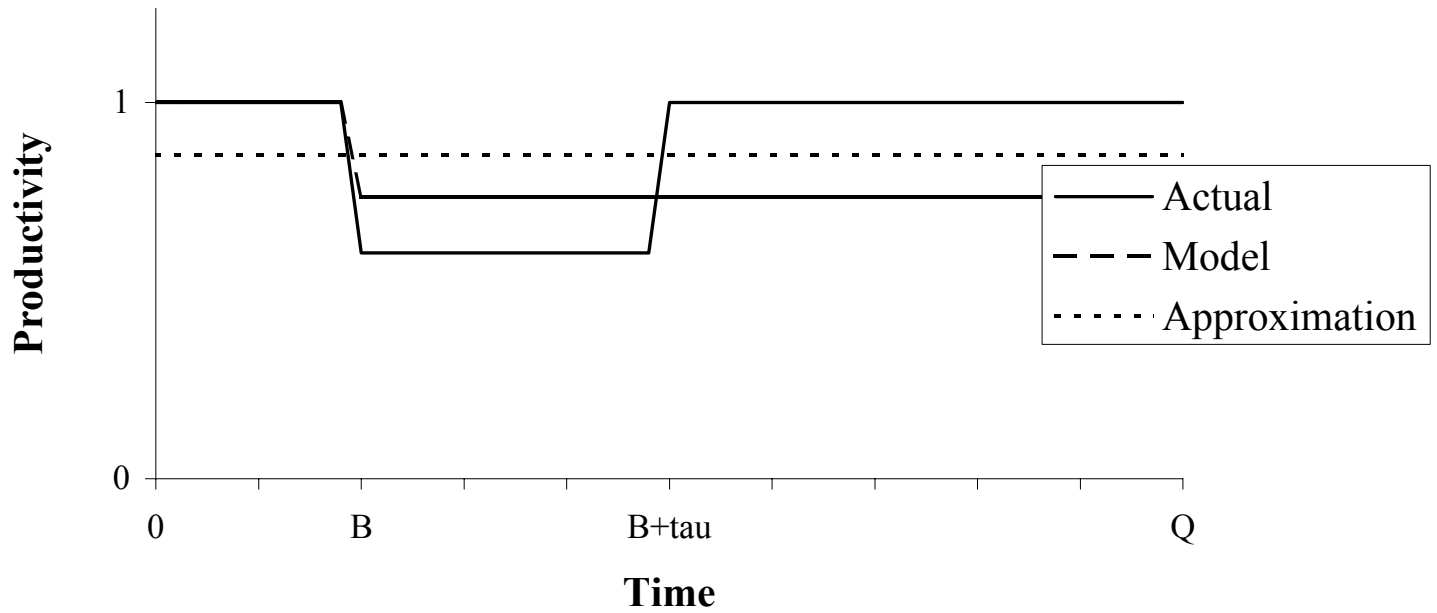




\section{Figure III \\ Average Lifetime Productivity as a \\ Function of Age at Childbearing}



Time of Childbearing

Figure IV

\section{Determination of Childbearing Age}

Solid circles indicate optimal childbearing ages.

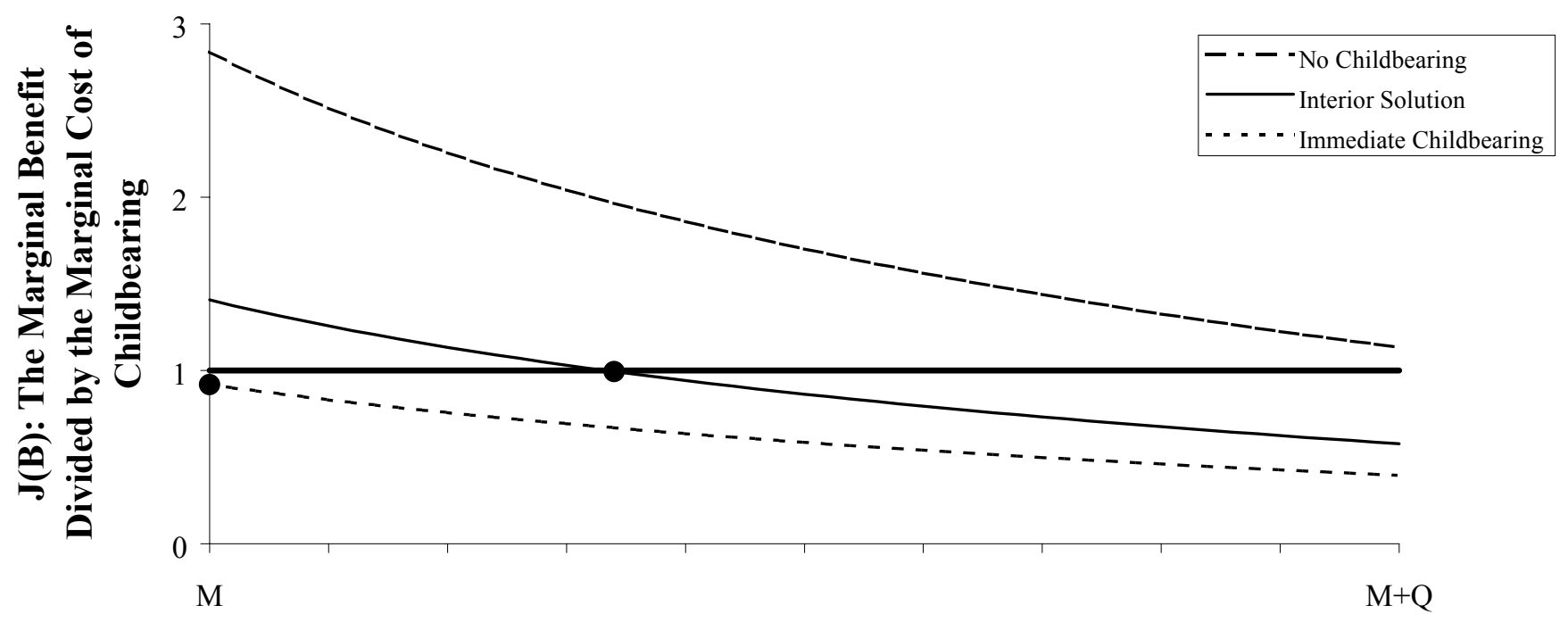

Age 


\section{Figure V}

\section{Optimal Paths for Human Capital and Human Capital Investment}

Based on a discrete-time version of the model and specific parameter values

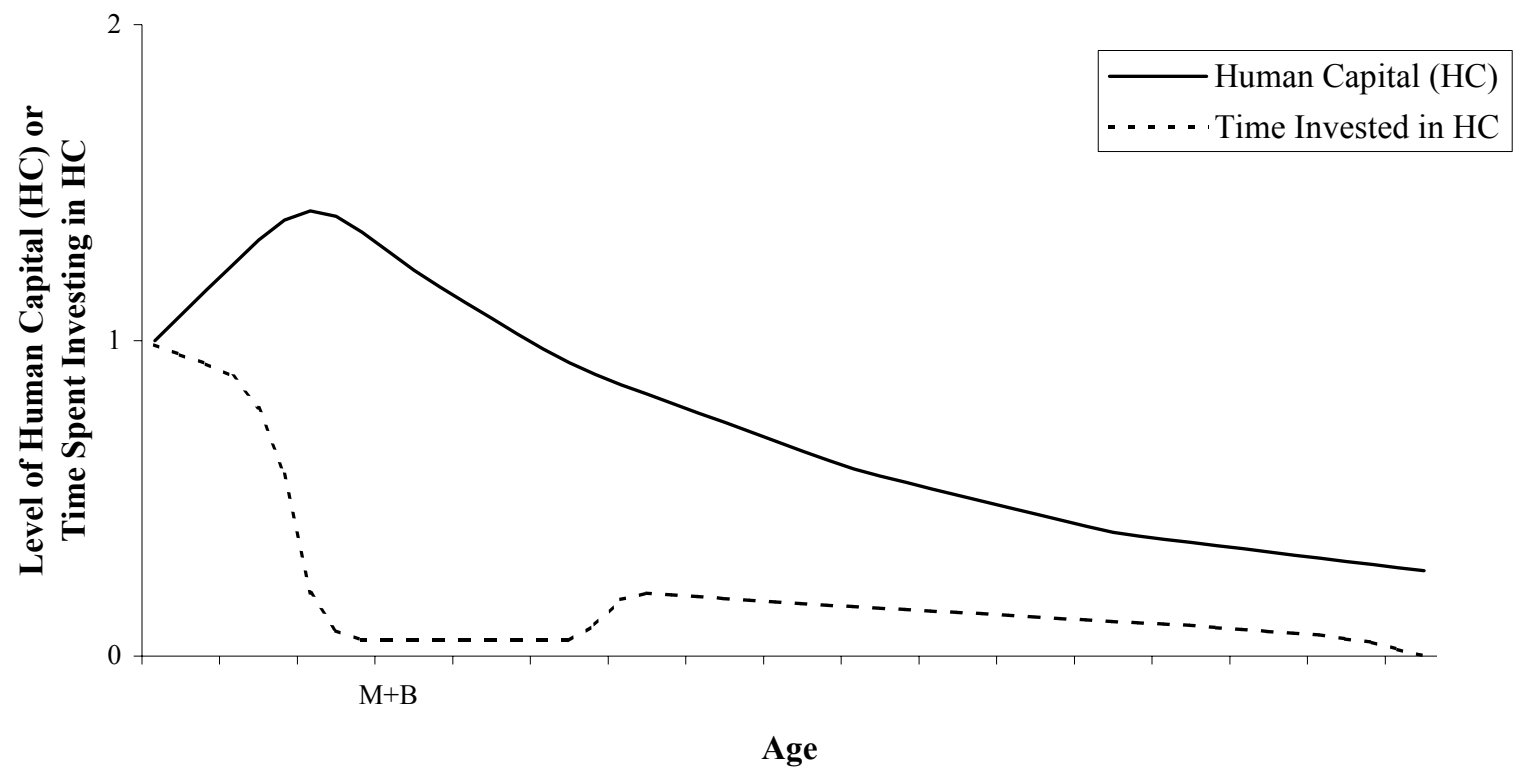

